\title{
Influência da filosofia no uso da tecnologia em enfermagem: revisão integrativa
}

Influence of philosophy in the use of nursing technology: integration review

Influencia de la filosofía en el uso de la tecnología en enfermado: revisión integrativa

Recebido: 05/03/2020 | Revisado: 09/03/2020 | Aceito: 11/03/2020 | Publicado: 22/03/2020

\section{Amanda Guimarães Ferreira}

ORCID: https://orcid.org/0000-0002-3680-8621

Universidade Federal do Rio Grande, Brasil

E-mail: ferreiraamandaguimaraes@gmail.com

Deciane Pintanela de Carvalho

ORCID: https://orcid.org/0000-0003-1598-6602

Universidade Federal do Rio Grande, Brasil

E-mail: deciane.carvalho@gmail.com

Edison Luiz Devos Barlem

ORCID: https://orcid.org/0000-0001-6239-8657

Universidade Federal do Rio Grande, Brasil

E-mail: ebarlem@gmail.com

\section{Luciano Garcia Lourenção}

ORCID: https://orcid.org/0000-0002-1240-4702

Universidade Federal do Rio Grande, Brasil

E-mail: luciano.famerp@gmail.com

Adriane Maria Netto de Oliveira

ORCID: https://orcid.org/0000-0001-9422-423X

Universidade Federal do Rio Grande, Brasil

E-mail: adrianenet@ vetorial.net

Laurelize Pereira Rocha

ORCID: https://orcid.org/0000-0001-9334-6550

Universidade Federal do Rio Grande, Brasil

E-mail: laurelize@gmail.com 


\title{
Resumo
}

Analisar a influência da filosofia no uso de tecnologia na enfermagem. Trata-se de uma revisão integrativa, que teve como questão norteadora "Como a filosofia influencia no uso da tecnologia na enfermagem?". A coleta de dados ocorreu em julho de 2017 nas bases de dados: Literatura LatinoAmericana e do Caribe em Ciências da Saúde, Scientific Electronic Library Online, Cumulative Index to Nursing and Allied Health Literature e International Medical Literature Analysis and Retrieval System Online via PubMed. A busca foi realizada por meio dos descritores: Filosofia em enfermagem, Tecnologia e Equipe de enfermagem e após os critérios de inclusão e exclusão, fizeram parte dos resultados 12 artigos. A enfermagem busca conhecimento científico para a utilização de tecnologias para a realização do cuidado, tanto por meio de equipamentos, programas de computador, quanto de tecnologias como as terapias alternativas, educação em saúde, entre outros. A produção científica evidencia diferentes formas de utilizar as tecnologias na enfermagem e a sua importância na prática de cuidados e assistência aos pacientes com qualidade.

Palavras-chave: Filosofia em Enfermagem; Tecnologia; Equipe de Enfermagem.

\begin{abstract}
To analyze the influence of philosophy on the use of technology in nursing. This is an integrative review, whose fundamental question was "How does philosophy influence the use of technology in nursing?". Data collection took place in July 2017 in the databases: Latin American and Caribbean Literature in Health Sciences, Electronic Scientific Library Online, Cumulative Literature Index in Nursing and Allied Health and International System of Analysis and Recovery of Medical Literature Online via PubMed. A research was carried out through the descriptors: Philosophy in nursing, Technology and Nursing team and after inclusion and exclusion, including part of the results 12 articles. A scientific research in nursing to use technologies to perform care, both for medium-sized equipment, computer programs, as well as technologies such as alternative therapies, health education, among others. Scientific production shows different forms of use as technologies in nursing and its importance in the practice of quality care and assistance to patients.
\end{abstract}

Keywords: Philosophy, Nursing; Technology; Nursing, Team.

\section{Resumen}

Analizar la influencia de la filosofía en el uso de la tecnología en enfermería. Se trata de una revisión integradora, cuya pregunta fundamental fue "Cómo influye la filosofía en el uso de la tecnología en enfermería?". La recolección de datos tuvo lugar en julio de 2017 en las bases de datos: Literatura latinoamericana y caribeña en ciencias de la salud, Biblioteca científica electrónica en línea, Índice de literatura acumulada en enfermería y salud aliada y Sistema internacional de análisis y recuperación de literatura médica. En línea a través de PubMed. Se realizó una búsqueda utilizando los descriptores: 
Filosofía en enfermería, Tecnología y equipo de enfermería y después de la inclusión y exclusión, incluyendo parte de los resultados 12 artículos. Investigación científica en enfermería para utilizar tecnologías en la provisión de atención, tanto para equipos medianos, programas informáticos y tecnologías como terapias alternativas, educación para la salud, entre otros. La producción científica muestra diferentes formas de uso como tecnologías en enfermería y su importancia en la práctica de atención y asistencia de calidad a los pacientes.

Palabras clave: Filosofía en Enfermería; Tecnología; Grupo de Enfermería.

\section{Introdução}

O termo tecnologia na saúde é atribuído ao uso de máquinas e equipamentos durante o processo de cuidar, no entanto, a tecnologia não deve ser vista apenas como resultado de ações em busca de melhorias para o tratamento de cuidado em saúde. A tecnologia também pode se manifestar por meio de conhecimento e habilidades ligados a utilização de recursos, de forma com que o cuidado seja individual, ético, seguro e humano (Sá Neto \& Rodrigues, 2010).

O avanço tecnológico baseia-se em produtos, serviços e na forma como as pessoas pensam a tecnologia, na enfermagem o uso da tecnologia evidencia a maneira como o indivíduo entende um fenômeno, a relação do mundo, seu significado e conceito, em hospitais de alta complexidade, serviços comunitários, ensino ou pesquisa (Melo et al., 2013).

A tecnologia expressa o conhecimento científico e sua transformação enquanto ciência, a qual necessita de intervenção ética, com isso a filosofia apresenta papel importante, possibilitando que o profissional reflita de forma crítica e participativa em relação ao seu fazer, além de conduzir o desenvolvimento científico responsável ${ }^{(3,4)}$. (Dantas, Santos \& Tourinho, 2016; Koerich et al., 2006).

A enfermagem deve estar instrumentalizada acerca de conhecimentos científicos quanto à utilização, riscos e beneficio do uso de tecnologias, bem como, o toque e o apoio emocional. Desta forma, destaca-se que a tecnologia e cuidados são constituídos como métodos que se complementam e estão interligados com o objetivo de manter a vida dos indivíduos (Schwonke et al., 2011).

Com isso, o uso das tecnologias é uma forma do enfermeiro prestar cuidado humanizado, buscando melhorias na qualidade de vida, comprometendo-se com sua prática, aprimorando seus conhecimentos para atuar na promoção, proteção e recuperação da saúde (Dantas, Santos \& Tourinho, 2016). Enfermeiros, docentes e estudantes de enfermagem 
devem utilizar a tecnologia como forma de desenvolver habilidade de pensamento crítico, com exigência de um olhar cuidadoso e ético, no local de trabalho e no ambiente de inserção do paciente (Melo et al., 2013).

A tecnologia também evidencia saberes e maneiras de cuidar, o que torna necessário a atualização de aperfeiçoamento dos profissionais de saúde para aplicação de conhecimento de maneira responsável e racional, com o desenvolvimento de senso crítico e reflexivo, repensando novas maneiras de cuidar, por meio da utilização da criatividade, sensibilidade e humanização das tecnologias (Sá Neto \& Rodrigues, 2010).

O bom uso da tecnologia pode tornar a vida menos frágil e complicada e mais prazerosa, para isso é imprescindível reflexão ética sobre responsabilidade, uma vez que os profissionais da saúde são agentes e produtores de técnicas, porém a utilização da tecnologia na tarefa de cuidar está associada a atitudes, gestos, palavras e experiências (Koerich et al., 2006).

Na prática da enfermagem é preciso compreender a importância do equilíbrio entre a tecnologia e o papel da equipe de enfermagem, tanto científico, quanto tecnológico e humanizado. Diversas tecnologias, equipamentos e processos de cuidados são capazes de desumanizar a assistência, por isso a tecnologia deve ser processada no pensamento e experiência dos profissionais, evidenciando que a tecnologia e cuidado humano são realidades constantemente construídas (Melo et al., 2013).

Desta forma, é necessário que os profissionais de enfermagem sejam capazes de realizar as técnicas de trabalho, assim como, sejam críticos no exercício da assistência, com competência e conhecimentos que permitam a compreensão e reflexão do trabalho em saúde, autonomia e capacidade para resolver problemas e comprometimento com a ética e a transformação da realidade (Dantas, Santos \& Tourinho, 2016).

Diante do exposto, surge o interesse em estudar a temática a partir dos conhecimentos adquiridos durante a disciplina de Filosofia da Ciência, da Saúde e da Enfermagem do curso de Doutorado em Enfermagem da Universidade Federal do Rio Grande, a qual motiva a busca por evidências científicas que demostrem a influencia da filosofia no uso das tecnologias na enfermagem. Desta forma, este estudo teve como objetivo analisar a influência da filosofia no uso de tecnologia na enfermagem. 


\section{Metodologia}

Trata-se de uma revisão integrativa desenvolvida por meio das seguintes etapas: 1) definição da questão norteadora; 2) determinação dos critérios de inclusão e exclusão; identificação das informações que serão extraídas dos artigos; 3) análise dos estudos selecionados; 4) interpretação dos resultados e 5) apresentação da revisão integrativa (Whittemore \& Knafl, 2005).

Como questão norteadora estabeleceu-se: Como a filosofia influencia no uso da tecnologia na enfermagem? Como critérios de inclusão optou-se por artigos científicos, publicados em todo o período de acesso das bases de dados selecionadas, com a finalidade de abranger o maior número de produção científica, nos idiomas português, inglês e espanhol, com disponibilidade de resumo e texto na íntegra online e gratuitamente. Os critérios de exclusão foram teses e dissertações.

A coleta de dados ocorreu em julho de 2017 nas bases de dados Literatura LatinoAmericana e do Caribe em Ciências da Saúde (LILACS), Scientific Electronic Library Online (SciELO), Cumulative Index to Nursing and Allied Health Literature (CINAHL) e International Medical Literature Analysis and Retrieval System Online (MEDLINE) via PubMed.

A busca foi realizada por meio dos termos: Filosofia em enfermagem, Tecnologia e Equipe de enfermagem, disponíveis no Medical Subject Headings (MeSH), na List of Headings do CINAHL Information Systems e Descritores em Ciências da Saúde (DeCS) da Biblioteca Virtual em Saúde e utilizou-se o operador booleano AND para os cruzamentos, de acordo com a Tabela 1 . 
(CC BY 4.0) | ISSN 2525-3409 | DOI: http://dx.doi.org/10.33448/rsd-v9i4.3026

Tabela 1: Distribuição de número de publicações por base de dados e cruzamento dos descritores selecionados. Rio Grande do Sul, Brasil, 2017.

\begin{tabular}{ccc}
\hline Bases de & Filosofia em enfermagem AND & Philosophy, Nursing AND \\
dados & Tecnologia AND Equipe de & tecnology AND Nursing, Team \\
& Enfermagem &
\end{tabular}

$\begin{array}{ccc}\text { SCIELO } & 0 & 0 \\ \text { LILACS } & 1 & 0 \\ \text { CINAHL } & 0 & 2 \\ \text { PUBMED } & 0 & 27 \\ & 1 & 29\end{array}$

Total

Fonte: Elaborado pelos autores.

A coleta de dados nas bases de dados identificou 30 resultados, após aplicou-se os critérios de inclusão e exclusão, realizando-se leitura de 18 resumos e textos na íntegra, excluíram-se ainda 6 artigos que não responderam a questão norteadora. Desta forma, fizeram parte dos resultados 12 artigos, conforme apresentado na Figura 1.

Figura 1: Fluxograma de seleção dos artigos. Brasil, 2017

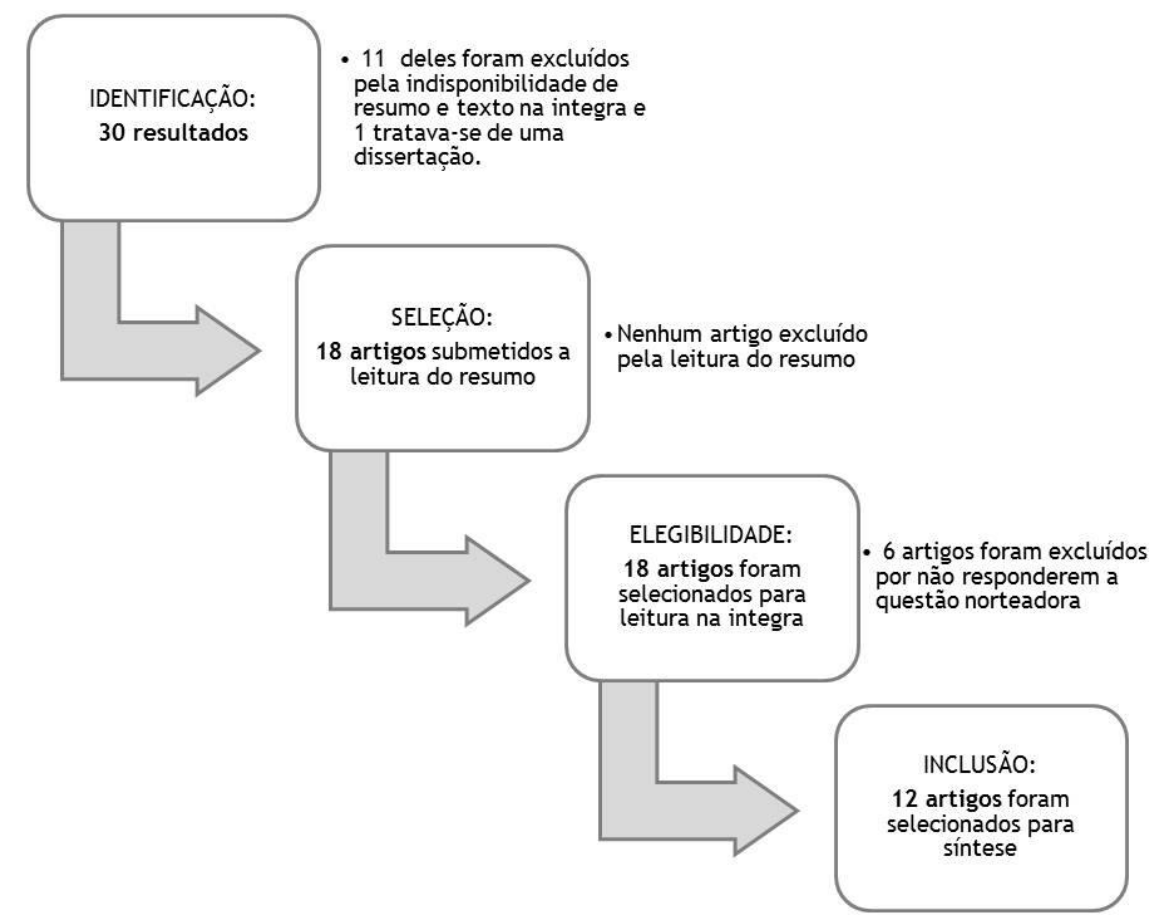

Fonte: Elaborado pelos autores. 
Para análise das publicações identificou-se o título do artigo, autores, periódico, ano de publicação, tipo de estudo e nível de evidência. O nível de evidência classifica as evidências quanto sua forma hierárquica e abordagem metodológica, como: nível 1 - revisão sistemática ou metanálise de múltiplos estudos clínicos randomizados; nível 2 - ensaios clínicos randomizados bem desenhados; nível 3 - ensaios clínicos bem desenhados sem randomização; nível 4 - estudos de caso-controle e de coorte; nível 5 - revisões sistemáticas de estudos descritivos e qualitativos; nível 6 - estudos descritivos ou de abordagem qualitativa; e nível 7 - opinião de especialistas (Melnyk \& Fineout-Overholt, 2005).

\section{Resultados}

Dos 12 artigos selecionados identificou-se que em 2016 ocorreu o maior número de publicações, com três artigos, seguido de 2015 com dois artigos. De acordo com a nacionalidade, 11 artigos foram publicados em periódicos internacionais e apenas um foi dos editado no Brasil. O nível de evidência que mais caracterizou a amostra foi o 5 contendo cinco artigos. No Quadro 1 apresenta-se uma síntese dos estudos analisados nesta revisão.

Quadro 1: Caracterização dos estudos segundo título, ano, periódico e nacionalidade, tipo de estudo e nível de evidência (NE). Brasil, 2017

\begin{tabular}{|c|c|c|c|c|c|}
\hline $\mathbf{N}^{\mathbf{0}}$ & Título & Ano & $\begin{array}{c}\text { Periódico/ } \\
\text { nacionalidade }\end{array}$ & Tipo de estudo & NE \\
\hline 01 & $\begin{array}{c}\text { Dare to be different: transformational } \\
\text { leadership may hold the key to } \\
\text { reducing the nursing shortage (Thyer, } \\
\text { 2003) }\end{array}$ & 2003 & $\begin{array}{c}\text { J Nurs Manag / } \\
\text { Internacional }\end{array}$ & Estudo de caso & 6 \\
\hline 02 & $\begin{array}{c}\text { Withdrawal of treatment in the } \\
\text { intensive care unit: the ethical and } \\
\text { legal issues (Allen-Smyth, 2001) } \\
\text { into health care education: a cautious } \\
\text { approach (Richardson, 2001) }\end{array}$ & 2001 & $\begin{array}{c}\text { Contemp Nurse. } \\
\text { / Internacional }\end{array}$ & Estudo de caso & 6 \\
\hline 04 & $\begin{array}{c}\text { Governing the conduct of conduct: are } \\
\text { restraints inevitable? (Irving, 2002) }\end{array}$ & 2002 & $\begin{array}{c}\text { J Clin Nurs. / } \\
\text { Internacional } \\
\text { Internacional }\end{array}$ & $\begin{array}{c}\text { Opinião de } \\
\text { especialistas }\end{array}$ & 7 \\
\hline 05 & $\begin{array}{c}\text { Enfermagem e genética: uma crítica } \\
\text { Enting complementary therapies }\end{array}$ & 2006 & $\begin{array}{c}\text { Estudos } \\
\text { Estudo caso }\end{array}$ & 6 \\
\hline
\end{tabular}


Research, Society and Development, v. 9, n. 4, e156943026, 2020

(CC BY 4.0) | ISSN 2525-3409 | DOI: http://dx.doi.org/10.33448/rsd-v9i4.3026

\begin{tabular}{|c|c|c|c|c|c|}
\hline & $\begin{array}{l}\text { feminista rumo ao trabalho em equipes } \\
\text { transdisciplinares (Anderson, Monsen } \\
\text { \& Rorty, 2006) }\end{array}$ & & $\begin{array}{c}\text { Feministas. / } \\
\text { Brasileiro }\end{array}$ & especialistas & \\
\hline 06 & $\begin{array}{l}\text { Involvement of the Hospice Family } \\
\text { Caregiver in Care Plan Meetings: } \\
\text { Mixed Methods Controlled and } \\
\text { randomized testing (Oliver et al., } \\
\text { 2017) }\end{array}$ & 2016 & $\begin{array}{c}\text { American } \\
\text { Journal of } \\
\text { Hospice \& } \\
\text { Palliative } \\
\text { Medicine/ } \\
\text { Internacional }\end{array}$ & $\begin{array}{c}\text { Estudo } \\
\text { randomizado }\end{array}$ & 1 \\
\hline 07 & $\begin{array}{l}\text { A New Patient Care Strategy for } \\
\text { Nursing, Health and Wellbeing } \\
\text { (Purnell \& Whitt, 2016) }\end{array}$ & 2016 & $\begin{array}{l}\text { Holistic nursing } \\
\text { practice/ } \\
\text { Internacional }\end{array}$ & $\begin{array}{c}\text { Revisão } \\
\text { sistemática }\end{array}$ & 5 \\
\hline 08 & $\begin{array}{l}\text { A systematic review of interventions to } \\
\text { change staff care practices in order to } \\
\text { improve resident outcomes in nursing } \\
\text { homes (Low et al., 2015) }\end{array}$ & 2015 & $\begin{array}{c}\text { PLoS One/ } \\
\text { Internacional }\end{array}$ & $\begin{array}{c}\text { Revisão } \\
\text { sistemática }\end{array}$ & 5 \\
\hline 09 & $\begin{array}{l}\text { A multimethod analysis of shared } \\
\text { decision-making in hospice } \\
\text { interdisciplinary team meetings } \\
\text { including family caregivers } \\
\text { (Washington et al., 2016) }\end{array}$ & 2016 & $\begin{array}{c}\text { Palliative } \\
\text { Medicine/ } \\
\text { Internacional }\end{array}$ & $\begin{array}{c}\text { Estudo } \\
\text { qualitativo }\end{array}$ & 5 \\
\hline 10 & $\begin{array}{l}\text { To what extent do primary care nurses } \\
\text { act as lifestyle coaching for case } \\
\text { managers in relation to weight } \\
\text { management? A systematic review } \\
\text { (Sonja, Dillen \& Hiddink, 2014) }\end{array}$ & 2014 & $\begin{array}{l}\text { BMC Fam } \\
\text { Pract/ } \\
\text { Internacional }\end{array}$ & $\begin{array}{c}\text { Revisão } \\
\text { sistemática }\end{array}$ & 5 \\
\hline 11 & $\begin{array}{l}\text { Efficiency achievements from a user- } \\
\text { developed real-time modifiable clinical } \\
\text { information system (Bishop, Patrick \& } \\
\text { Besiso, 2015) }\end{array}$ & 2015 & $\begin{array}{l}\text { Ann Emerg } \\
\text { Med. / } \\
\text { Internacional }\end{array}$ & $\begin{array}{l}\text { Opinião de } \\
\text { especialistas }\end{array}$ & 7 \\
\hline 12 & $\begin{array}{l}\text { Establishing a super-link system: } \\
\text { spinal cord injury rehabilitation } \\
\text { nursing (Chen \& Boore, 2007) }\end{array}$ & 2007 & $\begin{array}{l}\text { J Adv Nurs. / } \\
\text { Internacional }\end{array}$ & $\begin{array}{c}\text { Estudo } \\
\text { qualitativo }\end{array}$ & 5 \\
\hline
\end{tabular}




\section{Discussão}

A enfermagem é responsável por assumir o cuidado à beira do leito, no entanto, com o crescente aumento das tecnologias, especialmente nas unidades de cuidado intensivo, as enfermeiras passaram a buscar conhecimento científico para a utilização de máquinas, ventiladores, monitores, entre outros, como forma de fortalecer sua prática. Esse aspecto está ligado à característica da disciplina da enfermagem ter sido desenvolvida em uma filosofia de ciência que busca fontes de conhecimento e maneiras de desenvolvê-lo tendo como base o entendimento holístico do ser humano (Anderson, Monsen \& Rorty, 2006).

Os enfermeiros são agentes de mudança, participam no processo de aprendizagem do paciente e advogam pelos direitos dos pacientes e da profissão. A enfermagem é definida por exercer a liderança em transformar os cuidados de saúde, tendo em vista que os enfermeiros são identificados como visionários, criativos, com autonomia, capazes de realizar tomada de decisão com relação aos pacientes, além de possuírem qualidades voltadas a estratégias de comunicação ligadas à saúde (Thyer, 2003). Tais aspectos podem estar relacionados a influencia da filosofia no modo como a enfermagem presta os cuidados de enfermagem, os quais podem representar tecnologias em saúde.

Estudo com equipe multidisciplinar, envolvendo medicina, fisioterapia e enfermagem buscou discutir o uso de restrição em pacientes psiquiátricos e suas questões éticas. O uso de restrições é identificado com uma tecnologia de normalização utilizada em pessoas incapazes de se autogovernar, que ocorre por meio de uma observação hierárquica, por julgamentos e exames, produzindo um corpo dócil, o qual não é levado em consideração os argumentos para a sua liberdade. A equipe é responsável pela tomada de decisão sobre o uso de restrição, por meio da problematização das ideias, considerando acesso aos serviços e prioridade de atendimento, enfatizando a natureza complexa da prática de cuidados (Irving, 2002).

As questões éticas e legais também são discutidas em unidades de terapia intensiva em virtude de a sua configuração ser caracterizada pelas tecnologias, como o uso de máquinas para os cuidados de saúde, com isso, deve-se considerar também a retirada de tratamento nessas unidades e os princípios, doutrinas e legislação relacionadas a essa tomada de decisão, a qual pode ter como base uma estrutura ética para auxiliar neste processo (Allen-Smyth, 2001).

Nas unidades de terapia intensiva a enfermeira é parte vital da equipe multidisciplinar e realiza os cuidados aos pacientes mesmo que o tratamento médico seja suspenso, por meio dos cuidados paliativos. A tomada de decisão da equipe quanto à retirada de cuidados envolve 
(CC BY 4.0) | ISSN 2525-3409 | DOI: http://dx.doi.org/10.33448/rsd-v9i4.3026

a equipe, o paciente e a família, considerando as questões éticas frente ao direito a vida e a um tratamento inútil para prolongar a vida, crenças e desejos dos pacientes e familiares, e o enfermeiro atuam como advogado do paciente para a resolução de problemas por meio de uma comunicação efetiva para essa tomada de decisão (Allen-Smyth, 2001).

Outras formas de tecnologia em saúde são as terapias alternativas, esse aspecto é abordado no estudo que propõe a integração de terapias complementares na educação para profissionais de saúde, por meio da introdução de diferentes modalidades terapêuticas e suas respectivas questões filosóficas (Richardson, 2001). A utilização de terapias complementares como acupuntura, homeopatia, aroma terapia, massagens, entre outras tem sido desenvolvidas em ambientes de saúde como uma escolha entre os pacientes nos casos de ineficácia da medicina tradicional e aumento da disponibilidade dessas tecnologias.

Entretanto, as terapias complementares devem ser repensadas e utilizadas em integração com o tratamento convencional para a saúde, da mesma forma, os currículos de enfermagem necessitam ser adequados para que as terapias complementares sejam uma competência padrão das enfermeiras, podendo aconselhar sobre as intervenções em saúde (Richardson, 2001).

A tecnologia vem sendo utilizada cada vez mais como aliada na assistência à saúde por meio de diferentes formas de utilização. Dois estudos internacionais realizados em ambiente hospitalar psiquiátrico abordaram a temática ao utilizar a tecnologia para conectar cuidadores de pacientes psiquiátricos a equipe de saúde durante reuniões de plano de cuidado (Oliver et al., 2017; Washington et al., 2016).

As reuniões foram realizadas por conferências via web ou pelo telefone, onde os cuidadores e familiares poderiam expor suas dúvidas e sua opinião para a equipe de cuidados. O envolvimento do paciente/família nas decisões resultou em uma maior reflexão sobre o processo de cuidado na equipe, além de um plano de cuidado muito mais apropriado a cada paciente. $\mathrm{O}$ envolvimento de familiares e cuidadores em reuniões de equipe interdisciplinar usando tecnologia de videoconferência cria uma plataforma útil para a tomada de decisões compartilhadas (Oliver et al., 2017; Washington et al., 2016).

Outro estudo abordou a questão da bioletrodinâmica, que desempenha um importante papel na função biofísica e biológica de todos os organismos vivos. A partir de 1990, foram desenvolvidas ferramentas moleculares e celulares com a adição da nanotecnologia essas inovações tecnológicas, permitem a promoção da saúde e do bem estar de forma holística (Purnell \& Whitt, 2016). 
A pesquisa sobre estes novos dispositivos é realizada por uma equipe interdisciplinar, composta por biólogos, enfermeiros, bioestatisticos, engenheiros elétricos e inventores, que buscam através de ensaios clínicos trazerem medicamentos de medicina energética válidos. A natureza holística desta pesquisa, que tem seus fundamentos na física quântica, tem um grande potencial e deve ser vista como uma nova fronteira na qual a enfermagem pode assumir a liderança e pioneira como prática futura de medicina energética (Purnell \& Whitt, 2016).

Os sistemas de informação clínica também são tecnologias que estão cada vez mais ganhando espaço nas organizações de saúde, já que seu papel é fundamental para a precisão, agilidade e segurança nas informações. Estudo realizado no departamento de emergência de um hospital referência na Austrália comparou a eficiência entre dois sistemas utilizados no departamento tendo como objetivo assegurar um excelente atendimento ao paciente e sua segurança (Bishop, Patrick \& Besiso, 2015).

Para alcançar o objetivo, utilizaram a participação dos usuários e equipe clínica no processo de construção do sistema, onde seis novas funções foram projetadas: o gráfico de sinais vitais, perguntas frequentes do paciente, resumo dos cuidados, formas de triagem, entrega de exames e as tarefas de enfermagem. A tecnologia veio para assegurar um melhor trabalho para a equipe bem como um melhor cuidado para o paciente, principalmente por ter tido a participação dos profissionais no processo, tendo eles o conhecimento necessário para reconhecer as necessidades nas intervenções (Bishop, Patrick \& Besiso, 2015).

Um estudo realizado no Reino Unido teve como objetivo descrever o papel dos enfermeiros de cuidados primários e sua cooperação com outros profissionais no gerenciamento de doenças crônicas. Para isso, utilizaram uma revisão sistemática, que constatou a importância dos enfermeiros como uma grande ferramenta de auxilio nesse processo, sendo que os estudos mostraram que o enfermeiro tem um tempo maior do que outros profissionais para dar atenção necessária aos pacientes (Sonja, Dillen \& Hiddink, 2014).

Os profissionais além da prática de cuidados de rotina utilizam uma nova tecnologia para o cuidado com as doenças crônicas, entre eles foi identificado: o aconselhamento para um estilo de vida preventivo, a educação para o paciente e sua família e o monitoramento dos resultados. Além disso, o número de consultas com enfermeiros de cuidados primários foram significativamente maiores do que as realizadas por médicos, comprovando a importância do papel do enfermeiro nesse processo de cuidado (Sonja, Dillen \& Hiddink, 2014). 
Outro estudo de revisão abordou intervenções para modificar o atendimento de pessoas que vivem em lares de idosos e resultou em muitos estudos pregando a mudança da filosofia ou aspectos culturais. Essas abordagens revelam a importância de novas tecnologias do cuidado como: o cuidado centrado na pessoa e o cuidado orientado para a emoção, conscientização e restauração (Low et al., 2015).

Foram identificados que $80 \%$ dos artigos da revisão utilizaram uma teoria para o planejamento das intervenções como: modelo de mudança de oito passos de Kotter, implementação de Kitson do quadro de práticas baseadas em evidências, a teoria da aprendizagem social de Bandura dentre outras, e assim as intervenções mudaram pelo menos um aspecto da prática de cuidado da equipe de saúde. Além disso, outras ferramentas como o treinamento, as reuniões, o suporte da instituição, foram facilitadores para o melhor atendimento no lar de idosos (Low et al., 2015).

A enfermagem como profissão, vem utilizando cada vez mais as tecnologias no seu processo de trabalho, como mostra o estudo desenvolvido em Taiwan, que tinha por objetivo identificar as necessidades dos clientes e familiares cuidadores, com lesão de medula espinhal durante sua reabilitação, bem como as estratégias que os enfermeiros utilizam para atender essas necessidades. Com isso, surgiu à teoria Super-link, que propõe o cuidado de enfermagem como um processo contínuo de construção de relações interpessoais e envolve um cuidado holístico de atenção centrada no paciente, estimulando o trabalho com equipe interdisciplinar através de cuidados colaborativos e promovendo a esperança aos pacientes e seus familiares (Chen \& Boore, 2007).

\section{Considerações finais}

Os resultados deste estudo revelam a importância das diferentes formas de tecnologias na enfermagem, e devido à eficiência que geram no trabalho estão sendo cada vez mais utilizadas pelos serviços de saúde. Da mesma forma, as tecnologias são fundamentais para o cuidado de saúde, mas para total eficiência precisam de equipes treinadas e com capacidade de reflexão para utilizarem essa ferramenta de maneira adequada, garantindo promoção e recuperação da saúde, evidenciando a influencia da filosofia no uso de tecnologias na enfermagem.

Conclui-se que a filosofia é a base para que as tecnologias voltadas ao cuidado, como a utilização de máquinas, sistemas de comunicação e terapias complementares, bem como o uso das habilidades e conhecimentos de enfermagem, sejam utilizadas por meio do raciocínio 
crítico para a tomada de decisão, levando o profissional a buscar uma prática de cuidados e assistência de qualidade ao paciente.

Destaca-se que são necessários mais estudos que relacionem a temática filosofia e uso de tecnologias na enfermagem, com vistas a proporcionar melhor compreensão de como o uso das tecnologias exigem o desenvolvimento de competências profissionais na equipe de enfermagem voltadas ao reconhecimento das necessidades de saúde dos pacientes. Como limitação deste estudo, evidencia-se a escassez de estudos indexados em bases de dados a partir do cruzamento dos descritores Filosofia em Enfermagem, Tecnologia e Equipe de Enfermagem, Philosophy, Nursing, Tecnology, Nursing, Team.

\section{Referências}

Allen-Smyth, J.K. (2001). Withdrawal of treatment in the intensive care unit: the ethical and legal issues. Contemp Nurse, 10(1-2):70-82. Disponível em: https://doi.org/ 10.5172/conu.10.1-2.70. Acesso em 05 mar 2020.

Anderson, G.W., Monsen, R.B., \& Rorty, M.V. (2006). Enfermagem e genética: uma crítica feminista rumo ao trabalho em equipes transdisciplinares. Estudos Feministas, 14(2): 423 443. Disponível em: https://doi.org/10.1590/S0104-026X2006000200006. Acesso em 05 mar 2020.

Bishop, R.O., Patrick, J., \& Besiso, A. (2015). Efficiency achievements from a userdeveloped real-time modifiable clinical information system. Ann Emerg Med, 65(2):133-142. Disponível em: http://doi.org/10.1016/j.annemergmed.2014.05.032. Acesso 05 mar 2020.

Chen, H.Y., \& Boore, J.R. (2007). Establishing a super-link system: spinal cord injury rehabilitation nursing. J Adv Nurs, 57(6):639-648. Disponível em:http://doi.org/10.1111/j.1365-2648.2006.04160.x. Acesso em 05 mar 2020.

Dantas, C.N., Santos, V.E.P., \& Tourinho, F.S.V. (2016). A consulta de enfermagem como tecnologia do cuidado à luz dos pensamentos de Bacon e Galimberti. Texto Contexto Enferm, 25(1):e2800014. Disponível em: https://doi.org/10.1590/0104-0707201500002800014. Acesso em: 05 mar 2020. 
Irving, K. (2002). Governing the conduct of conduct: are restraints inevitable? J Adv Nurs, 40(4):405-412. Disponível em: http://onlinelibrary.wiley.com/doi/10.1046/j.13652648.2002.02388.x/abstract. Acesso em 05 mar 2020.

Koerich, M.S., Backes, D.S., Scortegagna, H.M., Wall, M.L., Veronese, A.M., Zeferino, M.T., ... Santos, A. (2006). Tecnologias de cuidado em saúde e enfermagem e suas perspectivas filosóficas. Texto Contexto Enferm, 15 (Esp):178-185. Disponível em: http://www.scielo.br/pdf/tce/v15nspe/v15nspea22. Acesso em 05 mar 2020.

Low, L., Fletcher, J., Goodenough, B., Jeon, Y., Etherton-Beer, C., MacAndrew, M. \& Beattie, E. (2015). A systematic review of interventions to change staff care practices in order to improve resident outcomes in nursing homes. PLoS One, 10(11):e0140711. Disponível em: http://doi.org/10.1371/journal.pone.0140711. Acesso em 05 mar 2020.

Melnyk, B.M., \& Fineout-Overholt, E. (2005) Making the case for evidence-based practice. In: Melnyk, B.M., Fineout-Overholt, E. Evidence-based practice in nursing \& healthcare. A guide to best practice. Philadelphia: Lippincot Williams \& Wilkins. 3-24.

Melo, E.C., Oliveira, R.R., Zurita, R.C.M., Santos, S.S.C., \& Mathias, T.A.F. (2013). Technology of nursing and care to premature newborn: a reflection theory. J Nurs UFPE, 7(7):4782-4788. Disponível em: https://doi.org/10.5205/reuol.4656-38001-2SM.0707201327. Acesso em 05 mar 2020.

Oliver, D.P., Demiris, G., Washington, K., Kruse, R.L., \& Petroski, G. (2017). Hospice Family Caregiver Involvement in Care Plan Meetings: A Mixed-Methods Randomized Controlled Trial. Am J Hosp Palliat Care. 34(9):849-859. Disponível em: http://doi.org/10.1177/1049909116661816. Acesso 05 mar 2020.

Purnell, M.C., \& Whitt, M.A. (2016). A New Patient Care Strategy for Nursing, Health and Wellbeing. Holistic nursing practice, 1-9.

Richardson, J. (2001). Integrating complementary therapies into health care education: a cautious approach. J Clin Nurs, 10(6):793-798. Disponível em: 
http://onlinelibrary.wiley.com/doi/10.1111/j.1365-2702.2001.00554.x/full. Acesso em 05 mar 2020.

Sá Neto, J.A., \& Rodrigues, B.M.R.D. (2010). Tecnologia como fundamento do cuidar em neonatologia. Texto Contexto Enferm, 19(2): 372-377. Disponível em: https://doi.org/10.1590/S0104-07072010000200020. Acesso em 05 mar 2020.

Schwonke, C.R.G.B., Lunardi Filho, W.D., Lunardi, V.L., Santos, S.S.C., \& Barlem, E.L.D. (2011). Phylosophical perspectives about the use of tecnology in critical care nursing. Rev Bras Enferm, 64(1):189-192. Disponível em: http://doi.org/10.1590/s003471672011000100028. Acesso em 05 mar 2020.

Sonja, M.E., Dillen, V., \& Hiddink, G.J. (2014). To what extent do primary care nurses act as lifestyle coaching for case managers in relation to weight management? A systematic review. BMC Fam Pract, 15:197. Disponível em: https://doi.org/10.1186/s12875-014-0197-2. Acesso 05 mar 2020.

Thyer, G.L. (2003). Dare to be different: transformational leadership may hold the key to reducing the nursing shortage. J Nurs Manag, 11(2):73-79. Disponível em: http://onlinelibrary.wiley.com/doi/10.1046/j.1365-2834.2002.00370.x/abstract. Acesso em 05 $\operatorname{mar} 2020$.

Washington, K.T., Oliver, D.P., Gage, L.A., Albright, D.L., \& Demiris, G. (2016). A multimethod analysis of shared decision-making in hospice interdisciplinary team meetings including family caregivers. Palliative Medicine, 30(3):270- 278. Disponível em: http://doi.org/10.1177/0269216315601545. Acesso em 05 mar 2020.

Whittemore, R., \& Knafl, K. (2005). The integrative review: updated methodology. J Adv Nurs, 52(5).

\section{Porcentagem de contribuição de cada autor no manuscrito}

Amanda Guimarães Ferreira - 30\%

Deciane Pintanela de Carvalho - 30\%

Edison Luiz Devos Barlem - 10\% 
Research, Society and Development, v. 9, n. 4, e156943026, 2020

(CC BY 4.0) | ISSN 2525-3409 | DOI: http://dx.doi.org/10.33448/rsd-v9i4.3026

Luciano Garcia Lourenção - 10\%

Adriane Maria Netto de Oliveira - 10\%

Laurelize Pereira Rocha - 10\% 